\title{
The effect of selected sterilization methods on antibacterial activity of aqueous extract of herbal plants.
}

\begin{abstract}
The aim of this study was to compare selected sterilization methods to maintain high susceptibility of antibacterial activities of aqueous extracts of herbal plants. Autoclavesterilized Impregnated disk and Impregnated disk sterilized by Acrodisc syringe filter were embedded on Mueller-Hinton Agar (MHA) plates seeded with the respective test microorganisms. Among five extracts, Euphorbia hirta exhibited antibacterial activities. Autoclaving caused less damage to the antibacterial activities of the tested extract in comparison with syringe filtration.
\end{abstract}

Keyword: Antibacterial activity; Aqueous extract; Euphorbia hirta; Sterilization methods 